\begin{tabular}{|c|l|}
\hline Title & $\begin{array}{l}\text { Attenuated total reflection Fourier transform infrared spectroscopy study of the adsorption of organic contaminants on a } \\
\text { hydrogen-terminated Si(111) surface in air }\end{array}$ \\
\hline Author(s) & Ye, Shen; Ichihara, Taro; U osaki, Kohei \\
\hline Citation & $\begin{array}{l}\text { A pplied Physics Letters, 75(11), 1562-1564 } \\
\text { https://doi.org/10.1063/124755 }\end{array}$ \\
\hline Issue Date & 1999 \\
\hline Doc URL & http://hdl.handle.net/2115/5502 \\
\hline Rights & Copyright $\odot 1999$ A merican Institute of Physics \\
\hline Type & article \\
\hline File Information & APL 75-11.pdf \\
\hline
\end{tabular}

Instructions for use 


\title{
Attenuated total reflection Fourier transform infrared spectroscopy study of the adsorption of organic contaminants on a hydrogen-terminated $\mathrm{Si}(111)$ surface in air
}

\author{
Shen Ye, Taro Ichihara, and Kohei Uosaki ${ }^{\mathrm{a})}$ \\ Physical Chemistry Laboratory, Division of Chemistry, Graduate School of Science, Hokkaido University, \\ Sapporo 060, Japan
}

(Received 13 May 1999; accepted for publication 14 July 1999)

\begin{abstract}
The adsorption of organic contaminants on a hydrogen-terminated $\mathrm{Si}(111)$ surface was investigated using attenuated total reflection Fourier transform infrared spectroscopy. When the hydrogen-terminated $\mathrm{Si}(111)$ surface was exposed to dry air, the sharp $\mathrm{Si}-\mathrm{H}$ monohydride peak became weaker and a broad component became visible in the lower wave-number region. Furthermore, a number of bands within the $\mathrm{C}-\mathrm{H}$ stretching region were observed. The intensity of the sharp $\mathrm{Si}-\mathrm{H}$ band was recovered to a certain extent and those of the broad component and the $\mathrm{C}-\mathrm{H}$ bands decreased after the sample was rinsed in hexane. These results suggest that the contamination by organic adsorbates is not accompanied by a chemical bond formation. (C) 1999 American Institute of Physics. [S0003-6951(99)01337-6]
\end{abstract}

Contamination of Si surfaces by organic species significantly deteriorates the reliability of semiconductor device performance in ultra-large-scale integrated circuits (ULSIs). ${ }^{1,2}$ It has been reported that a trace amount of organic contaminants still exists on the Si surface ${ }^{3-5}$ even after Si wafers were cleaned using the RCA cleaning procedure. ${ }^{6}$ Attenuated total reflection Fourier transform infrared (ATRFTIR) spectroscopy has been widely applied to investigate the vibration state of organic adsorbates on Si surfaces, especially on the $\mathrm{Si}(100)$ surface. ${ }^{7-9}$ Although the study of the structure of adsorbates on $\mathrm{Si}(100)$ is important in view of practical application, quantitative investigation on this surface is difficult since there is no established technique to prepare an atomically flat $\mathrm{Si}(100)$ surface. Recently, the preparation of a well-defined $\mathrm{Si}(111)-(1 \times 1)$ surface terminated by a monohydride was realized by etching in a concentrated $\mathrm{NH}_{4} \mathrm{~F}$ solution. ${ }^{10}$ This ideal $\mathrm{H}-\mathrm{Si}(111)-(1 \times 1)$ surface provides an excellent model to study the chemical reaction process on a well-defined $\mathrm{Si}$ surface. Furthermore, although it is known that hydrogen termination $(\mathrm{Si}-\mathrm{H})$ protects the $\mathrm{Si}$ surface from contamination and oxidation, no quantitative study has yet been carried out to clarify its role.

In the present study, ATR-FTIR spectroscopy was employed to monitor the surface contamination process by organic contaminants on a well-defined $\mathrm{H}-\mathrm{Si}(111)$ surface during exposure to a dry air environment in real time. The nature of the adsorption state of the airborne organic contaminants was discussed based on the intensity and band shape of the bands of the $\mathrm{Si}-\mathrm{H}$ and $\mathrm{C}-\mathrm{H}$ stretching vibrations.

Parallelogram ATR prisms $\left(26 \times 20 \times 0.38 \mathrm{~mm}, 45^{\circ}\right.$ bevel angle) were prepared from floating-zone-grown Si(111) wafers (Shin-Etsu Semiconductor, $n$-type, 50-500 $\Omega \mathrm{cm}$, both sides polished) by polishing the side portions of the wafers with alumina powder (final size, $0.3 \mu \mathrm{m}$ ). The

${ }^{a)}$ Electronic mail: uosaki@pcl.sci.hokudai.ac.jp internal reflection number within the Si prism was $\sim 70$.

A $\mathrm{Si}(111)$ ATR prism was cleaned in a sonication bath of acetone and then with Milli-Q water (resistivity $>18 \mathrm{M}$ $\Omega \mathrm{cm}$ ), oxidized in a boiling solution of concentrated $\mathrm{HCl}: \mathrm{H}_{2} \mathrm{O}_{2}: \mathrm{H}_{2} \mathrm{O}$ (1:1:4) for $20 \mathrm{~min}$, etched in $40 \% \mathrm{NH}_{4} \mathrm{~F}$ solution (Morita Chemical Industries, EL grade) for $5 \mathrm{~min}$, sonicated in Milli-Q water for $5 \mathrm{~s}$, and finally, dried in flowing purified nitrogen gas. The sample was mounted on an ATR accessory (Pike Technology) immediately after the preparation process.

ATR-FTIR measurements were performed using a BioRAD FTS-30 spectrometer equipped with a $\mathrm{HgCdTe}$ (MCT) detector cooled with liquid nitrogen. The dry air was continuously provided to the FTIR chamber by a BioRAD air dryer (type II, dew-point $50{ }^{\circ} \mathrm{C}$ ) in which a heatless drier (HD-0.5, CKD) containing a molecular sieve $13 \times$ and an oil-free compressor (Hitachi 0.4-OP-7S, 45 1/min) were used. The ATR-FTIR spectra were recorded in $p$-polarization with respect to a spectrum of an oxidized $\mathrm{Si}(111)$ surface as a function of the time exposed in a dry air environment. All spectra were obtained by integrating 128 interferograms with a resolution of $0.5 \mathrm{~cm}^{-1}$. Deconvolution of the band was carried out using WIN-IR software (version 4.14, Galactic Industries Corporation) with a Lorentz function.

Figure 1(a) shows the ATR-FTIR spectra of a $\mathrm{Si}(111)$ surface in the regions of $2300-2000$ and $3000-2800 \mathrm{~cm}^{-1}$ after etching in $40 \% \mathrm{NH}_{4} \mathrm{~F}$ solution. A very sharp peak was observed at $2084 \mathrm{~cm}^{-1}$ [full width at half maximum (FWHM) $\left.\sim 1 \mathrm{~cm}^{-1}\right]$. This peak is attributed to the stretching vibration of the monohydride on the $\operatorname{Si}(111)-(1 \times 1)$ surface, whereas no bands related to the dihydride or trihydride were observed. This result is in good agreement with that reported by Higashi et al., ${ }^{10}$ demonstrating that an atomically flat $\mathrm{Si}(111)$ surface terminated by an extremely homogeneous monolayer of hydrogen was obtained. No band was found in the region of $3000-2800 \mathrm{~cm}^{-1}$.

Figures 1(b)-1(e) show the ATR-FTIR spectra obtained after various exposure times. As the exposure time increased, 


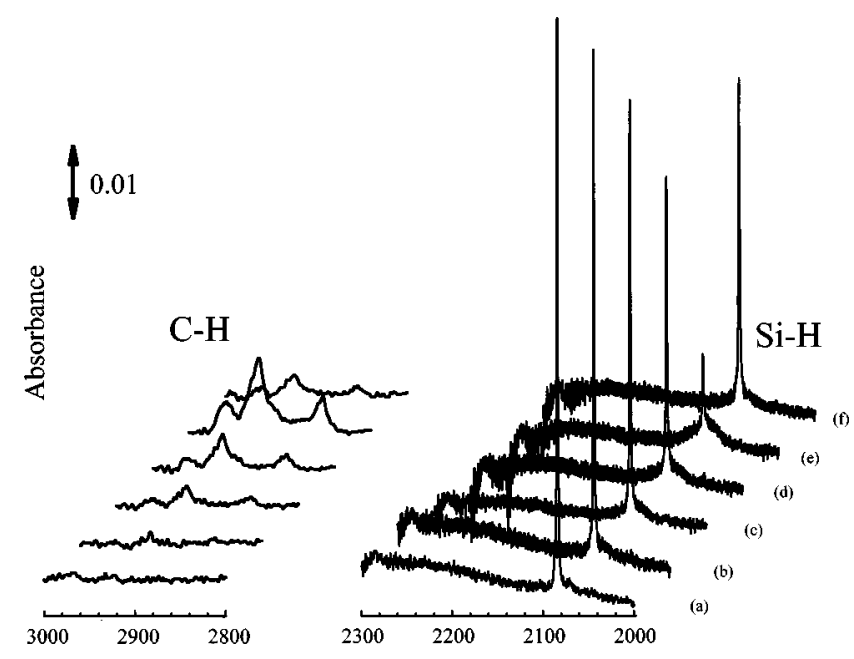

FIG. 1. ATR-FTIR spectra of a hydrogen-terminated Si(111) surface in the regions of $2300-2000 \mathrm{~cm}^{-1}$ and $3000-2800 \mathrm{~cm}^{-1}$ after the sample was exposed to dry air for (a) 0, (b) 8, (c) 24, (d) 48, and (e) $120 \mathrm{~h}$ and (f) was sonicated in hexane after (e).

the peak height of the sharp $\mathrm{Si}-\mathrm{H}$ band became smaller and a broad component became visible at the bottom of the $\mathrm{Si}-\mathrm{H}$ peak. At the same time, a number of bands grew in the $\mathrm{C}-\mathrm{H}$ stretching region of $3000-2800 \mathrm{~cm}^{-1}$. Three pronounced peaks at 2852, 2923, and $2960 \mathrm{~cm}^{-1}$ and a small shoulder at $2931 \mathrm{~cm}^{-1}$ were observed in this region. These peaks can be assigned to the symmetric $\left(2852 \mathrm{~cm}^{-1}\right)$ and asymmetric $\left(2923 \mathrm{~cm}^{-1}\right)$ stretching of methylene group and asymmetric stretching $\left(2960 \mathrm{~cm}^{-1}\right)$ and Fermi resonance $\left(2931 \mathrm{~cm}^{-1}\right)$ of the methyl group of hydrocarbon species adsorbed on the Si surface. ${ }^{11}$ The appearance of these bands shows the adsorption of organic contaminants. Similar bands were previously reported $^{7-9}$ and were attributed to organic contaminants introduced during the wafer cleaning process. ${ }^{7,8}$ In the present case, however, since no bands were observed in the $\mathrm{C}-\mathrm{H}$ region immediately after etching [Fig. 1(a)], contamination during the etching process can be excluded and these $\mathrm{C}-\mathrm{H}-$ related bands should arise from adsorbed hydrocarbon impurities which were contained in the dry air.

When the $\mathrm{Si}(111)$ sample was sonicated in hexane after a $120 \mathrm{~h}$ exposure to dry air, the intensities of the $\mathrm{C}-\mathrm{H}$ bands and the sharp $\mathrm{Si}-\mathrm{H}$ peak decreased and increased, respectively, as shown in Fig. 1(f). The recovery of the sharp Si-H peak after the sonication shows that the $\mathrm{Si}-\mathrm{H}$ band was not irreversibly decomposed.

To analyze the results more quantitatively, the intensities of the various bands were plotted as a function of time in Fig. 2. The $\mathrm{Si}-\mathrm{H}$ band was deconvoluted into two components, i.e., a sharp component at $2084 \mathrm{~cm}^{-1}$ with a FWHM of $\sim 1 \mathrm{~cm}^{-1}$ and a broad component that peaked at a slightly lower wave number $\left(2071 \mathrm{~cm}^{-1}\right)$ with a FWHM of $\sim 20$ $\mathrm{cm}^{-1}$. These two components were separately plotted. While the former peak decreased with time, the latter increased. The total intensity of these two bands also decreased with time but less significantly than the sharp peak shown in Fig. 2. The exposure time dependence of the intensity of the band due to the $\mathrm{C}-\mathrm{H}$ asymmetric stretch of the methylene group at $2923 \mathrm{~cm}^{-1}$ is also shown in Fig. 2 that represents the amount of adsorbed organic contaminants, which clearly increased with time. It is clear that the increase in the $\mathrm{C}-\mathrm{H}$ band is

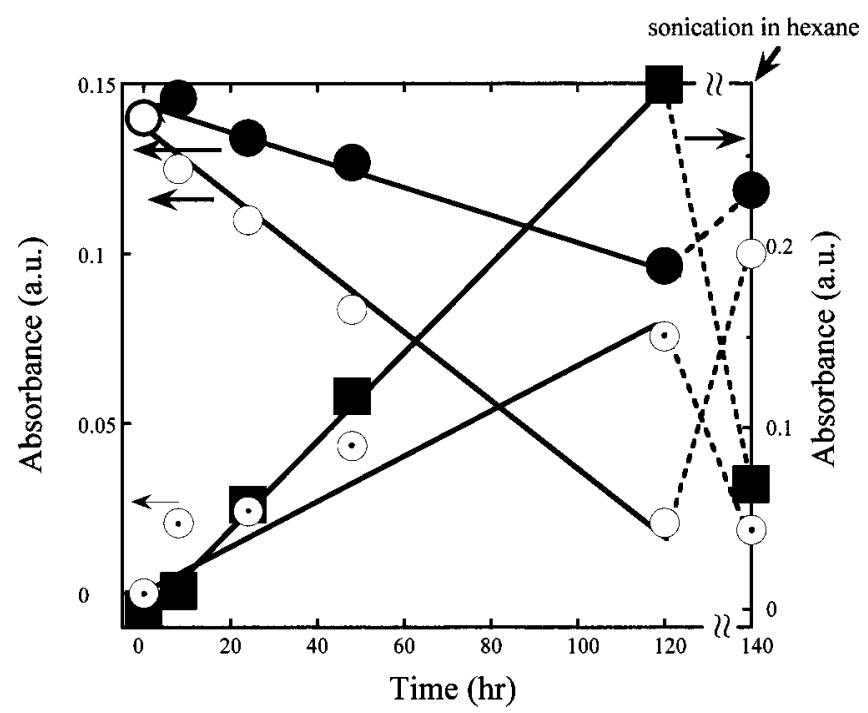

FIG. 2. Intensities of $\mathrm{Si}-\mathrm{H}$ and $\mathrm{C}-\mathrm{H}$ bands as a function of time and after the sonication in hexane. White, dotted, and black circles correspond to intensities of deconvoluted sharp, broad, and total $\mathrm{Si}-\mathrm{H}$ bands, respectively. The black square shows the intensity of the $\mathrm{C}-\mathrm{H}$ bands represented by the intensity of the band due to the $\mathrm{C}-\mathrm{H}$ asymmetric stretch of the methylene group at $2923 \mathrm{~cm}^{-1}$.

strongly correlated with the decrease and increase in the sharp and broad $\mathrm{Si}-\mathrm{H}$ bands, respectively.

The intensities of these bands after the sonication in hexane are also presented in Fig. 2. The recovery of the sharp $\mathrm{Si}-\mathrm{H}$ band after the sonication seems to be correlated with the decrease in the $\mathrm{C}-\mathrm{H}$ and broad $\mathrm{Si}-\mathrm{H}$ bands.

Two possibilities can be considered for the intensity change of the $\mathrm{Si}-\mathrm{H}$ bands. One is the oxidation of the $\mathrm{Si}(111)$ surface during the exposure to air. Niwano et al. actually reported the observation of a new band around 2250 $\mathrm{cm}^{-1}$, which was attributed to the formation of $\operatorname{SiH}\left(\mathrm{O}_{3}\right)$ after a hydrogen-terminated $\mathrm{Si}(111)$ surface was exposed to air with $10 \%$ humidity for longer than $\sim 50 \mathrm{~h}{ }^{12}$ In the present study, however, no peak was observed around 2250 $\mathrm{cm}^{-1}$ even after a 5 day exposure [Fig. 1(e)], showing that $\mathrm{SiH}\left(\mathrm{O}_{3}\right)$ was not formed on the surface. An x-ray photoelectron spectroscopy measurement also confirmed the absence of silicon oxide after the same exposure time. Thus, the decrease in the sharp $\mathrm{Si}-\mathrm{H}$ peak should not be due to the oxide formation. Oxide did not grow in the present study possibly because the $\mathrm{Si}(111)$ surface was exposed to only dry air. The hydrogen-terminated $\mathrm{Si}(111)$ seemed to effectively passivate the surface from oxidation in dry air.

The more likely reason is the adsorption of organic contaminants because there is a good correlation between the changes in the intensities of the $\mathrm{Si}-\mathrm{H}$ and $\mathrm{C}-\mathrm{H}$ bands. The effects of organic species on the $\mathrm{Si}-\mathrm{H}$ band have been investigated by various groups. Linford et al. reported that the $\mathrm{Si}-\mathrm{H}$ bands disappeared irreversibly after a chemically bonded organic monolayer was formed on the $\mathrm{Si}(111)$ surface. ${ }^{9}$ Since a significant portion of the sharp $\mathrm{Si}-\mathrm{H}$ band, which was lost during air exposure, was recovered by sonication in hexane, the loss of the $\mathrm{Si}-\mathrm{H}$ band was not irreversible in the present case. Dumas et al. observed that when $\mathrm{C}_{60}$ was deposited on a $\mathrm{Si}(111)$ surface, the $\mathrm{Si}-\mathrm{H}$ band was shifted to a lower wave number $\left(2062 \mathrm{~cm}^{-1}\right)$ and became broader (FWHM: $\sim 10 \mathrm{~cm}^{-1}$ ) while the total intensity of the 
$\mathrm{Si}-\mathrm{H}$ band did not change. They proposed that the van der Waals interaction between $\mathrm{C}_{60}$ and $\mathrm{Si}-\mathrm{H}$ caused the peak shift and broadening. ${ }^{13}$ Band broadening phenomena of $\mathrm{Si}-\mathrm{H}$ were also observed when the hydrogen-terminated $\mathrm{Si}(111)$ surface was in contact with aqueous ${ }^{14-16}$ and nonaqueous solutions. ${ }^{17}$ The fact, observed in the present study, that the peak position of the broad component of the $\mathrm{Si}-\mathrm{H}$ band shifted only slightly, the broad component grew as the sharp component decreased during the exposure to air, and the broad and sharp components decreased and increased, respectively, by the sonication in hexane, all suggest that the broad component is due to $\mathrm{Si}-\mathrm{H}$ interacting with some species and these two components are reversibly converted into each other. $\mathrm{C}-\mathrm{H}$ bands were also observed in the higher wave-number region than those of the chemically bonded monolayer, ${ }^{9}$ indicating a lower order of the organic adsorbate layer than that of the chemically bonded monolayer. Furthermore, the broad component increased in parallel to the increase in the $\mathrm{C}-\mathrm{H}$ bands. Thus, one can conclude that the broad component is due to the stretching vibration of $\mathrm{Si}-\mathrm{H}$ that has a strong interaction with physisorbed organic contaminants.

Based on the above results, one can explain the present results as follows. When a hydrogen-terminated $\mathrm{Si}(111)$ surface is exposed to air, organic contaminants are adsorbed on the surface because of the strong hydrophobic nature of the hydrogen-terminated $\mathrm{Si}(111)$ surface. As a result, the intensities of the $\mathrm{C}-\mathrm{H}$ bands increase. The organic contaminants seemed to form islands leaving other portions of the hydrogen-terminated surface clean because the position and the width of the sharp $\mathrm{Si}-\mathrm{H}$ band were not affected by the adsorption of the organic contaminants. Sonication in hexane removed most of the contaminants, leading to the increase in the free $\mathrm{Si}-\mathrm{H}$ area. As a result, the intensities of the broad and sharp components of the $\mathrm{Si}-\mathrm{H}$ band decrease and increase, respectively, and that of the $\mathrm{C}-\mathrm{H}$ related bands decreased.

One should note that the intensity of the sharp $\mathrm{Si}-\mathrm{H}$ bands did not recovered its original value and $\mathrm{C}-\mathrm{H}$ bands were still observed after the sonication in hexane. One of the reasons for this observation may be that the adsorbed organic contaminants are poorly soluble in hexane. The formation of a small amount of chemically bonded organic species and/or polymerized species may also contribute to the irreversible behavior of $\mathrm{Si}-\mathrm{H}$ on the $\mathrm{Si}(111)$ surface. Detailed investigations are now in progress.

The ATR-FTIR measurements clearly showed that the hydrogen-terminated $\mathrm{Si}(111)$ surface was not oxidized even after 5 days as long as the surface was exposed to only dry air. However, the surface was contaminated with the hydrocarbon impurities contained in dry air. Most of the contaminants can be removed by sonication in hexane.

This work was partially supported by a Grant-in-Aid for Scientific Research on Priority Area of "Electrochemistry of Ordered Interfaces"' (No. 09237101) from the Ministry of Education, Science, Sports and Culture, Japan. Kitazawa of Shin-Etsu Semiconductor is acknowledged for the donation of the Si wafers.

${ }^{1}$ K. Saga and T. Hattori, Appl. Phys. Lett. 71, 3670 (1997).

${ }^{2}$ T. Ogata, C. Ban, A. Ueyama, S. Muranaka, T. Hayashi, K. Kobayashi, J. Kobayashi, H. Kurokawa, Y. Ohno, and M. Hirayama, Jpn. J. Appl. Phys., Part 1 37, 2468 (1998).

${ }^{3}$ M. Grundner and H. Jacob, Appl. Phys. A: Solids Surf. 39, 73 (1986).

${ }^{4}$ A. Licciardello, O. Puglisi, and S. Pignataro, Appl. Phys. Lett. 48, 41 (1986).

${ }^{5}$ T. Takahagi, S. Shingubara, H. Sakaue, K. Hoshino, and H. Yashima, Jpn. J. Appl. Phys., Part 2 35, L818 (1996).

${ }^{6}$ W. Kern and D. A. Puotien, RCA Rev. 31, 187 (1970).

${ }^{7}$ L. Ling, S. Kuwabara, T. Abe, and F. Shimura, J. Appl. Phys. 73, 3018 (1993).

${ }^{8}$ O. M. R. Chyan, J. Chen, F. Xu, and J. Wu, Anal. Chem. 69, 2434 (1997).

${ }^{9}$ M. R. Linford, P. Fenter, P. M. Eisenberger, and C. E. D. Chidsey, J. Am. Chem. Soc. 117, 3145 (1995).

${ }^{10}$ G. S. Higashi, Y. J. Chabal, G. W. Trucks, and K. Raghavachari, Appl. Phys. Lett. 56, 656 (1990).

${ }^{11}$ N. B. Colthup, L. H. Daly, and S. E. Wiberley, Introduction to Infrared and Raman Spectroscopy, 3rd ed. (Academic, New York, 1990).

${ }^{12}$ M. Niwano, J. Kageyama, K. Kurita, K. Kinashi, I. Takahashi, and N. Miyamoto, J. Appl. Phys. 76, 2157 (1994).

${ }^{13}$ P. Dumas, M. Gruyters, P. Rudolf, Y. He, L. Yu, G. Gensterblum, R. Caudano, and Y. J. Chabal, Surf. Sci. 368, 330 (1996).

${ }^{14}$ L. M. Peter, D. J. Blackwood, and S. Pons, Phys. Rev. Lett. 62, 308 (1989).

${ }^{15}$ J. Rappich, H. J. Lewerenz, and H. Gerischer, J. Electrochem. Soc. 140, L187 (1993).

${ }^{16}$ M. Nakamura, M.-B. Song, and M. Ito, Electrochim. Acta 41, 681 (1996).

${ }^{17}$ F. Ozanam, A. Djebri, and J.-N. Chazalviel, Electrochim. Acta 41, 687 (1996). 